\title{
Synthetic Linemaps for Hierarchial Clouds
}

\author{
L. G. STENHOLM \\ Astronomical Observatory \\ Uppsala University \\ Box 515 \\ S 75120 Uppsala \\ Sweden \\ and \\ Swedish Defence Research Establishment \\ $S 17290$ Sundbyberg \\ Sweden
}

Molecular clouds are characterized by large density contrasts and pronounced internal motions. Some information on their internal physics has been acquired through relatively simple data analysis and through theoretical modelling, mainly hydrodynamical calculations. More detailed radiative transfer models have been limited to spherical cloud models.

A related but less recognized problem is the classification of molecular clouds, or otherwise stated, can we compress the information in the observed two-dimensional maps for various line parameters in such a way that the physical differences are retained, while the random component has been removed? This is a more fundamental formulation of the special problem of comparing the appearance of two molecular clouds, or of the problems of quantifying the velocity correlation within the clouds.

This paper addresses the question of radiative transfer in fully three dimensional cloud models, the problem is solved under the limiting condition of LTE. We have here concentrated our effort on the ${ }^{13} \mathrm{CO} \mathrm{J}=1-0$ line which is formed in LTE in the clouds.

The basic idea is to divide the cloud region in small cubic cells with constant physical conditions. The smallest possible scale-length of a fluctuation which can be represented by our model is thus the side length of such a cell. The largest possible scale-length is naturally the size of the cloud region. There are no limitations on the variations of the physical conditions between two neighbouring cell.

The cloud is organised in a number of generations of fragments, with identical physical conditions for all fragments of the same order. The velocity field is divided in two parts, the microscopic velocity field and the macroscopic velocity field. A number of different velocity fields and their combinations have been tried;

1. Each fragment moves through space with the velocity specified by eq. 2 .

2. The fragments rotates.

3. Radial expansion or contraction is assumed.

4. The velocity field is formed by a spectrum of Alfven waves.

The line parameters is defined in the standard way but note that no separation in line components is done. Each model produces of the order of 10000 spectra. The number of independent units of information in the model spectra is thus around 25 (spectral range covered divided by the width of the thermal profile). The total information content per model is thus typically 250000 information units.

The data analysis therefore rests on two postulates: 
1. The total information pool contains true information on the physical processes, as well as "noise" due to physical causes which are well represented by the chaos concept.

2. It is possible to reduce the data, without prior information on the physics, in such a way that the essence of true information is retained in a few parameters, and to eliminate the noise in the process.

The first reduction step is to compress the frequency information by the introduction of the standard line parameters. The line parameters used $\left(T_{B}, V\right.$ and $\left.D v\right)$ can be regarded as the lowest order moments of the intensity distribution in a spectra. The integrated intensity is a measure of the total number of photons (energy) in a spectrum.

The maximum amount of information retained after this step is 40000 units.

We can approach the spatial compression by using an analogy with the frequency case. The spatial information in a line parameter map is strongly influenced by chance, relative distances of fragments will depend for ex. on line of sight and starting conditions for the evolution. The spatial correlation information is thus ignored and one retains only the statistical distribution (i.e. number of elements per line parameter interval). The statistical moments for the distribution of each of line parameters can now be determined.

We have now reduced the information content to 12 parameters for each model (three moments for each line parameter). The cluster analysis is now used in the final step of the analysis to determine the multidimensional distance between any two models and to group similar models together.

If the method is successful each cluster should contain clouds which are in some way physically similar.

The separation according to important physical parameters is found to be very good, though not totally efficient. The calculations presented here indicates that the method is most sensitive to changes in the velocity field and least sensitive to the details of how the fragments are defined. These results can probably be explained by noting that changes in the velocity field influences 3 of the four line parameters (T, V and Dv) in contrast to density and abundance structures which mainly influences the peak intensity and the integrated intensity. The specifications of fragment distribution finally influences all line parameters but only indirectly through the velocity and density variations connected with the various fragment orders. The method presented is thus obviously capable of compressing substantial physical information on the cloud into a few characteristics and of eliminating most of the random component, but its sensitivity probably varies for the various physical parameters determining the cloud physics.

The cluster analysis show that models 47,48 and 52 are closest to the observed B5 cloud (e.g. the three models and B5 belong to the same cluster). All three model clouds are basically wave cloud models but models 48 and 52 have a small translational component in the velocity field. The multidimensional distance to B5 is larger for model 47 than for the other two.

Models 48 and 52 are characterized by a relatively small density gradient and by a whole spectrum of interfering "chaotic" waves, the amplitudes of the waves are independent of frequency (for simplicity) and the velocity does not depend on density. It was impossible to find a way to modify the hierarchical models so that they could be brought in closer agreement with the B5 characteristics. The major stumbling block is the line width. All hierarchical models tried have two problems, large regions with small $\Delta \mathrm{v}$ and very chaotic line profiles in contradiction to the observed line profiles. It may be possible to bring the hierarchical models in reasonable agreement with $B 5$ if one postulates that all mass is contained in the highest order fragments. An example is model 6, which has a multi-dimesional distance to the B5 cloud which is comparable to the distances of the most successful models.

The models computed cover a relatively small part of the possible range of parameters and this work is in that sense rather exploratory, even though the modelling were quite extensive. Any conclusions on the physics of the B5 cloud can thus be premature, but the conclusions given above seems to be rather independent of the model details. 